\title{
Vector boson and Charmonium production in proton-lead and lead-lead collisions with ATLAS at the LHC
}

\section{Zvi Citron*广}

Weizmann Institute of Science (IL)

E-mail: zvi.citronecern.ch

\begin{abstract}
Electroweak bosons do not interact strongly with the dense and hot medium formed in nuclear collisions, and thus should be sensitive to the nuclear modification of parton distribution functions (nPDFs). The in-medium modification of heavy charmonium states plays an important role in studying the hot and dense medium. The ATLAS detector, optimized to search for new physics in proton-proton interactions, is well equipped to measure $\mathrm{Z}$ and $\mathrm{W}$ bosons as well as quarkonium in the high occupancy environment produced in heavy ion collisions. Results from the ATLAS experiment on $\mathrm{W}$ and $\mathrm{Z}$ boson yields as a function of centrality, transverse momentum and rapidity, in lead-lead and proton-lead collisions are presented. Quarkonium results from proton-lead interactions are also shown.
\end{abstract}

38th International Conference on High Energy Physics

3-10 August 2016

Chicago, USA

\footnotetext{
* Speaker.

${ }^{\dagger}$ For the ATLAS Collaboration
} 


\section{Introduction}

Electroweak (EW) bosons are a valuable tool to study the hot and dense medium created in heavy-ion collisions. Since they are insensitive to the strong force and do not interact with the QCD medium, the rate of their production in different centrality classes, allows a test of binary collision scaling within heavy ion collisions. The production of $\mathrm{EW}$ bosons in $\mathrm{Pb}+\mathrm{Pb}$ collisions has been found to be proportional to the mean number of binary collisions present in the ion-ion interaction $[1,2]$. This finding confirmed the applicability of pQCD calculations in heavy-ion collisions, as well as the Glauber model description of the nucleonic geometry of the collisions. The experimental precision of these measurements, however, did not reach the level needed in order to determine whether there are nuclear modifications of the parton distribution function (PDF). The $\mathrm{p}+\mathrm{Pb}$ collision system is more sensitive to the effects of nuclear modification of the PDF, and the larger dataset obtained by the ATLAS experiment [3] from the 2013 LHC run enabled greater experimental precision in the results making it a fertile system to study.

Similarly, even as a full understanding of charmonium and bottomonium production in $\mathrm{A}+\mathrm{A}$ collisions remains elusive, 'Cold Nuclear Matter' effects have been observed in $p(d)+A$ interactions. The study of quarkonium in $\mathrm{p}+\mathrm{Pb}$ collisions is informative both as a baseline for $\mathrm{A}+\mathrm{A}$ collisions and as an avenue to study nuclear modification.

\section{Electroweak Bosons in Proton-Lead Collisions}

The cross-section of $\mathrm{Z}$ bosons is measured via decays in the di-muon and di-electron channels [4], and the $\mathrm{W}$ boson cross-section measured in the muon + neutrino channel [5] by the ATLAS experiment in $\mathrm{p}+\mathrm{Pb}$ collisions at $5.02 \mathrm{TeV}$. Figure 1 shows the rapidity differential $\mathrm{Z}$ boson cross section and the $\mathrm{W}$ boson cross-section differentially in the decay muon pseudorapidity. The data are compared to pQCD models based on the CT10 PDF [6] calculated at NLO, and show significant deviation from those models. The $\mathrm{Z}$ boson data is also compared to a model, EPS09 [7] that incorporates nuclear modification to the CT10 PDF. Including nuclear modification of the PDF in the calculation produces the correct description of the shape of the rapidity differential cross section (we note that although the shape of the model matches the data the total cross-section is lower than the data; newer calculations at NNLO using an updated PDF, CT14, remedy this overall under-prediction in the pQCD calculations). These data suggest that the EW bosons measured by ATLAS in $\mathrm{p}+\mathrm{Pb}$ collisions are sensitive to nuclear modification of the PDF.

\section{Quarkonium in Proton-Lead Collisions}

The yield of $\mathrm{J} / \Psi$ has been measured in $\mathrm{p}+\mathrm{Pb}$ collisions at $5.02 \mathrm{TeV}$ by the ATLAS experiment via their di-muon decay channel. Using the reconstructed mass and proper time of the di-muon pair, the observed $\mathrm{J} / \Psi$ candidates may be identified as prompt or non-prompt as well as distinguishing between the ground $\mathrm{J} / \Psi$ state and the excited $\Psi(2 \mathrm{~S})$ state. To gauge nuclear effects in the crosssection measured in $\mathrm{p}+\mathrm{p}$ collisions we use the nuclear modification factor. The nuclear modification factor, $\mathrm{R}_{p P b}$, is defined as the ratio of the cross sections where the $\mathrm{p}+\mathrm{p}$ cross section has been scaled 

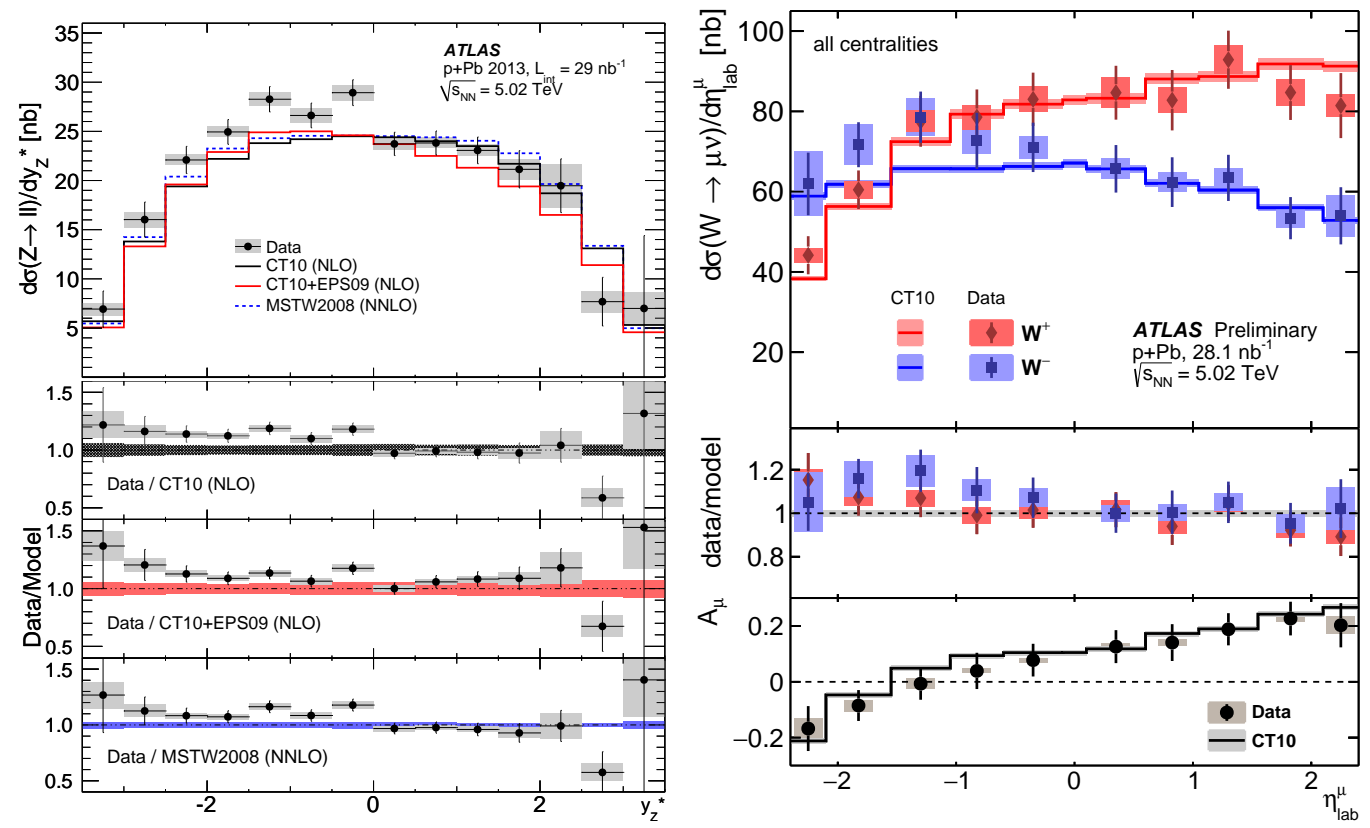

Figure 1: Left: The $\mathrm{d} \sigma / \mathrm{dy}$ distribution from $Z \rightarrow \ell \ell$, shown along with several model calculations in the upper panel. The lower three panels show the ratios of the data to the models. [4] Right: The upper panel shows the cross section of $W^{+}$(diamonds) and $W^{-}$(squares) as a function of the muon pseudorapidity and for a model (lines) constructed using CT10 PDFs. The middle panel shows the data-to-model ratios. The lower panel shows the asymmetry $\mathrm{A}_{\ell}=\left(\mathrm{N}_{W^{+}}-\mathrm{N}_{W^{-}}\right) /\left(\mathrm{N}_{W^{+}}+\mathrm{N}_{W^{-}}\right)$compared to the model. [5] The bars indicate statistical uncertainty and the shaded boxes systematic uncertainty. An additional $2.7 \%$ luminosity uncertainty on the cross section is not shown in either figure.

by $\mathrm{A}_{P b}(=208)$ :

$$
R_{p P b}=\frac{1}{A_{P b}} \frac{d^{2} \sigma_{p P b} / d y d p_{T}}{d^{2} \sigma_{p p} / d y d p_{T}} .
$$

The nuclear modification factor as a function of rapidity for prompt $\mathrm{J} / \Psi$, non-prompt $\mathrm{J} / \Psi$, and prompt $\Psi(2 S)[8]$ is shown in Figure 2, and a similar plot for upsilon [9] in Figure 3.

The data show consistency with unity indicating no observation of nuclear modification relative to $\mathrm{p}+\mathrm{p}$ collisions.

\section{Centrality in Proton-Lead Collisions}

For a more detailed understanding of the EW boson and quarkonium production cross-sections measured in $\mathrm{p}+\mathrm{Pb}$ collisions, we consider the yields differentially in collision centrality. In order to characterize the $\mathrm{p}+\mathrm{Pb}$ collision geometry, each event is assigned a centrality based on the total transverse energy measured in the forward calorimeter on the Pb-going side $(-4.9<\eta<-3.2)$. The standard Glauber model [10] approach is used to calculate the mean number of participating nucleons, $\left\langle N_{\text {part }}\right\rangle$. The mean number of inelastic nucleon-nucleon collisions, $\left\langle N_{\text {coll }}\right\rangle$, is $\left\langle N_{\text {part }}\right\rangle-1$. In addition, a Glauber-Gribov Color Fluctuation (GGCF) model, an extension to the Glauber model which allows event-by-event fluctuations of the nucleon-nucleon cross section $\sigma(N+N \rightarrow X)$, is 

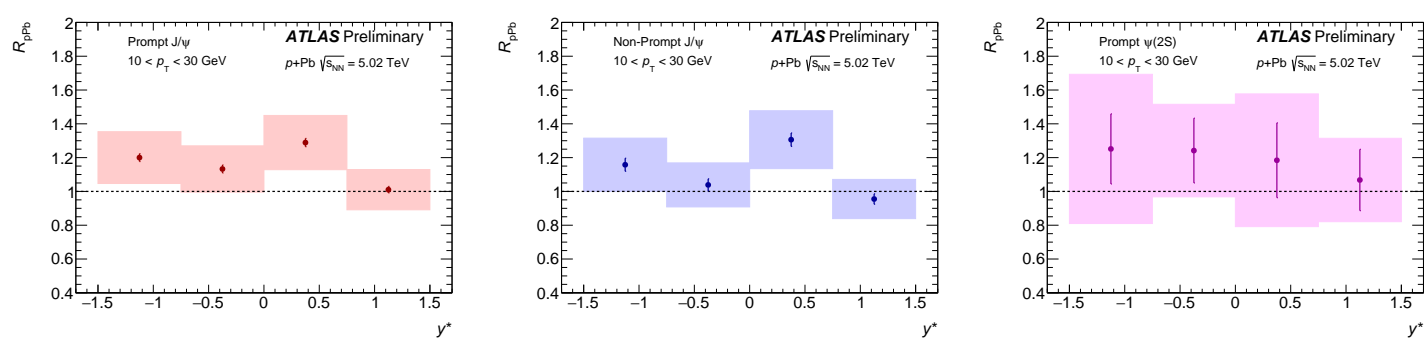

Figure 2: The nuclear modification factor, $\mathrm{R}_{p P b}$, as a function of rapidity for the production of prompt $\mathrm{J} / \Psi$ (left), non-prompt $\mathrm{J} / \Psi$ (middle) and prompt $\Psi(2 \mathrm{~S})$ (right). The error bars indicate only the statistical uncertainties, and the boxes represent the systematic uncertainties. [8]

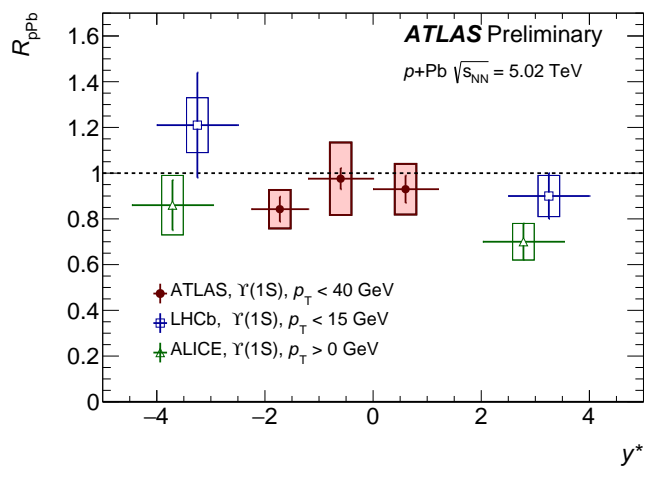

Figure 3: The nuclear modification factor $\mathrm{R}_{p P b}$ for $\Upsilon(1 \mathrm{~S})$ as a function of rapidity for $\mathrm{p}+\mathrm{Pb}$ collisions at $5.02 \mathrm{TeV}$. The vertical error bars indicate the statistical uncertainties, and the vertical open boxes indicate the systematic uncertainties. [9]

also considered. In this model the magnitude of the fluctuations is characterized by the parameter $\omega_{\sigma}$, with $\omega_{\sigma}=0$ corresponding to the standard Glauber model. The centrality models are discussed at greater length in [4]. Figure 4 shows the production of $\mathrm{Z}$ bosons for different centrality classes. The left panel shows that the yield scales approximately with the $\left\langle N_{\text {coll }}\right\rangle$ only if a 'centrality bias correction' [11] is applied or the GGCF model with $\omega_{\sigma}=0.11$ is used. Further, the right panel shows that the modification of the rapidity differential cross-section shown in Figure 1 is more pronounced in central collisions compared to peripheral collisions. Similarly, Figure 5 shows that the deviations from the model in the differential $\mathrm{W}$ boson yield displayed in Figure 1 is also centrality dependent. Together these suggest that there is some centrality dependence of the nuclear PDF modification.

To avoid ambiguities about the definition of centrality, we may use the $\mathrm{Z}$ boson yield as a 'standard candle' for centrality and examine the yields of other observables relative to the $\mathrm{Z}$ boson yield. Figure 6 shows the ratio of prompt $\mathrm{J} / \Psi$ and $\Psi(2 \mathrm{~S})$ production to $\mathrm{Z}$ boson production as a function of the energy deposited in the $\mathrm{Pb}$-going side forward calorimeter. The figure shows that the yield of $\mathrm{J} / \Psi$ as a function of centrality mirrors that of the $\mathrm{Z}$ boson, however the $\Psi(2 \mathrm{~S})$ is relatively suppressed in more central events.

This research is supported by the Israel Science Foundation (grant 1065/15) and by the 

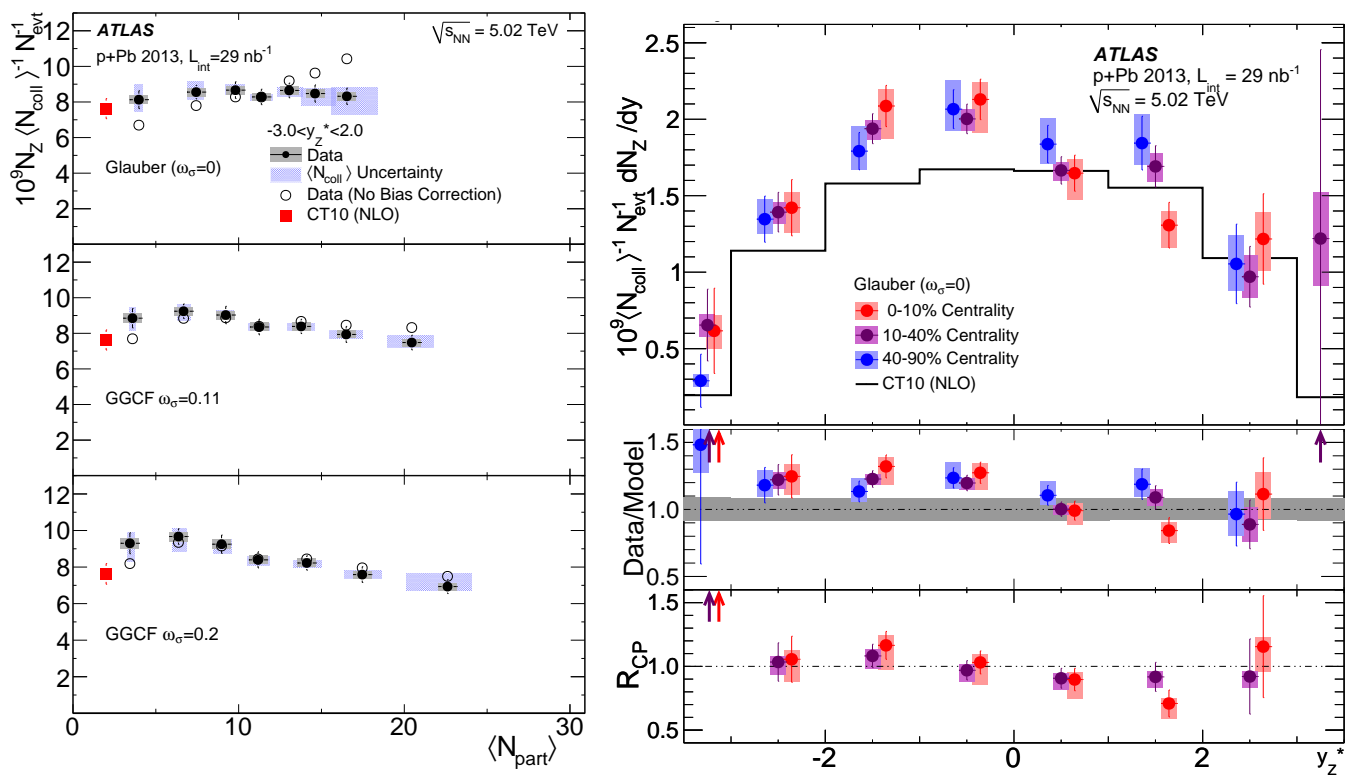

Figure 4: Left:The yield of $\mathrm{Z}$ bosons per event scaled by the mean number of nucleon-nucleon collisions $\left\langle N_{\text {coll }}\right\rangle$ as a function of the mean number of participating nucleons $\left\langle N_{\text {part }}\right\rangle$. Each panel uses a different Glauber model configuration in calculating $\left\langle N_{\text {part }}\right\rangle$ (and $\left\langle N_{\text {coll }}\right\rangle$ ). The data are compared to the CT10 model prediction plotted at $\left\langle N_{\text {part }}\right\rangle=2$. The bars indicate statistical uncertainty and the shaded boxes systematic uncertainty. As a reference, the data are plotted as they would be with no centrality bias correction in the open points (see text). Right: The top panel is the rapidity differential $\mathrm{Z}$ boson yields, scaled by $\left\langle N_{\text {coll }}\right\rangle$, for three centrality ranges compared with the CT10 model calculation. The middle panel shows the ratios of the data to the model. The bottom panel shows the ratio of the two more central yields to the peripheral 40-90\% yield.[4]

MINERVA Stiftung with the funds from the BMBF of the Federal Republic of Germany.

\section{References}

[1] ATLAS Collaboration, Phys. Rev. Lett. 110, (2013) 022301

[2] ATLAS Collaboration, Eur. Phys. J. C (2015) 75: 23

[3] ATLAS Collaboration, JINST 3 (2008) S08003

[4] ATLAS Collaboration, Phys. Rev. C92, 044915 (2015)

[5] ATLAS Collaboration, ATLAS-CONF-2015-056 (2015) https://cdsweb.cern.ch/record/2055677

[6] H.-L. Lai, M. Guzzi, J. Huston, Z. Li, P. M. Nadolsky, J. Pumplin, and C.-P. Yuan, Phys. Rev. D82 (2010) 074024

[7] H. Paukkunen and C. A. Salgado, JHEP 1103 (2011) 071

[8] ATLAS Collaboration, ATLAS-CONF-2015-023 (2015) https://cdsweb.cern.ch/record/2029577

[9] ATLAS Collaboration, ATLAS-CONF-2015-050 (2015) https://cdsweb.cern.ch/record/2055266

[10] M. L. Miller, K. Reygers, S. J. Sanders, and P. Steinberg, Ann. Rev. Nucl. Part. Sci. 57 (2007) 205-243

[11] D. V. Perepelitsa and P. A. Steinberg, arXiv:1412.0976 [nucl-ex] 

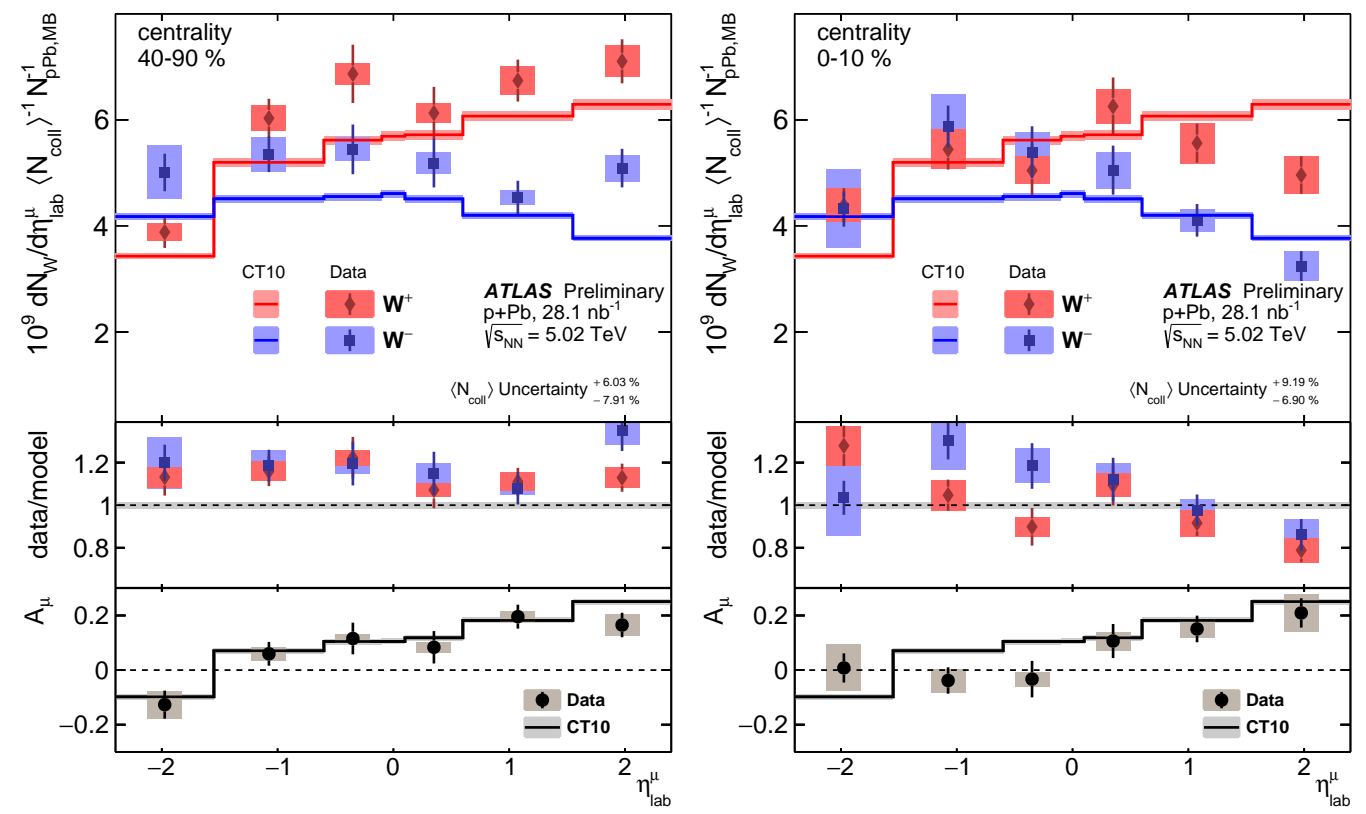

Figure 5: The upper panels show the production rate per nucleon-nucleon collision and per minimum-bias events taken in the corresponding centrality class of the $\mathrm{W}$ boson as function of the muon pseudorapidity. Diamonds show the results for $\mathrm{W}^{+}$and squares for $\mathrm{W}^{-}$. Results are compared to the POWHEG-based model using CT10 PDFs and the ratio of neutrons and protons in Pb nuclei (dashed lines). The data-tomodel ratios are shown in the middle panels for the particles of both charges. The lower panels show the asymmetry defined by $\mathrm{A}_{\ell}=\left(\mathrm{N}_{W^{+}}-\mathrm{N}_{W^{-}}\right) /\left(\mathrm{N}_{W^{+}}+\mathrm{N}_{W^{-}}\right)$compared to the same model. The left figure is for $40-90 \%$ centrality and the right for $0-10 \%$. [5]
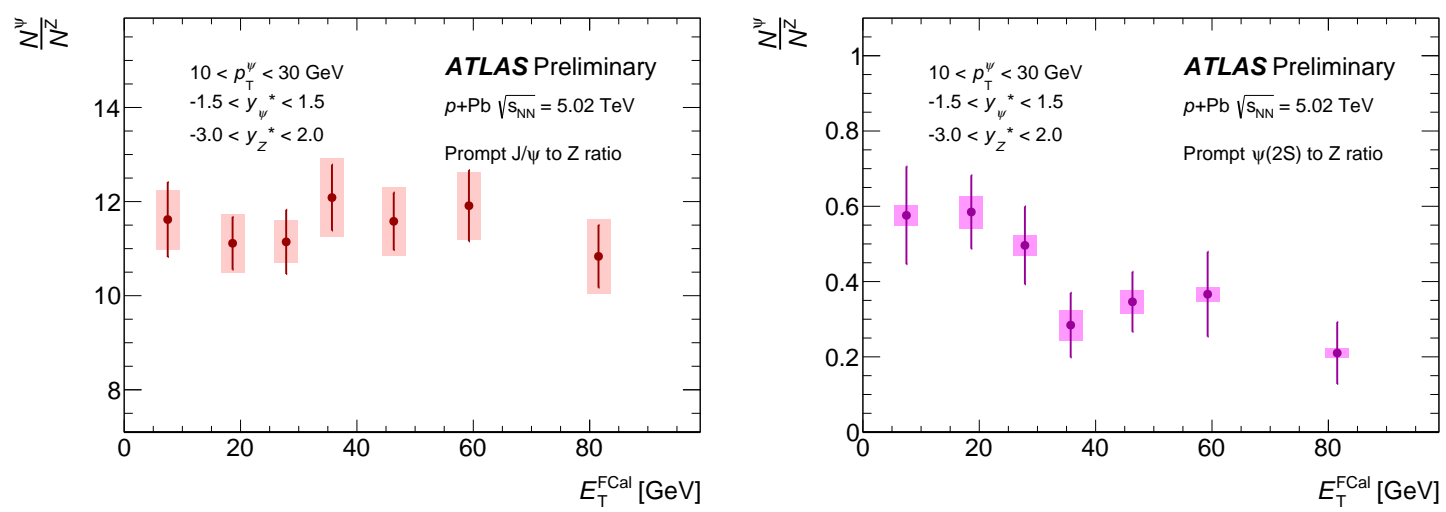

Figure 6: The $\mathrm{J} / \Psi$ yield to $\mathrm{Z}$ yield ratio as a function of the energy deposited in the forward calorimeter representing the centrality of the event, for the production of prompt J/ $\Psi$ (left), and prompt $\Psi$ (2S) (right). The error bars indicate only the statistical uncertainties, and the boxes represent the systematic uncertainties. [8] 